\title{
Candidiasis caused by Candida kefyr in a neonate: Case report
}

\author{
Stefan Weichert ${ }^{1,5^{*}}$, Konrad Reinshagen ${ }^{2}$, Katrin Zahn², Gernot Geginat ${ }^{3}$, Annebärbel Dietz ${ }^{3}$, Anna Kristina Kilian ${ }^{4}$, \\ Horst Schroten ${ }^{1}$ and Tobias Tenenbaum ${ }^{1}$
}

\begin{abstract}
Background: Systemic Candidia infections are of major concern in neonates, especially in those with risk factors such as longer use of broad spectrum antibiotics. Recent studies showed that also term babies with underlying gastrointestinal or urinary tract abnormalities are much more prone to systemic Candida infection. We report a very rare case of candidiasis caused by Candida kefyr in a term neonate.

Case Presentation: Renal agenesis on the left side was diagnosed antenatally and anal atresia postnatally. Moreover, a vesico-ureteral-reflux (VUR) grade $V$ was detected by cystography. The first surgical procedure, creating a protective colostoma, was uneventful. Afterwards our patient developed urosepsis caused by Enterococcus faecalis and was treated with piperacillin. The child improved initially, but deteriorated again. A further urine analysis revealed Candida kefyr in a significant number. As antibiotic resistance data about this non-albicans Candida species are limited, we started liposomal amphotericin B (AMB), but later changed to fluconazole after receiving the antibiogram. Candiduria persisted and abdominal imaging showed a Candida pyelonephritis. Since high grade reflux was prevalent we instilled AMB into the child's bladder as a therapeutic approach. While undergoing surgery (creating a neo-rectum) a recto-vesical fistula could be shown and subsequently was resected. The child recovered completely under systemic fluconazole therapy over 3 months.
\end{abstract}

Conclusions: Candidiasis is still of major concern in neonates with accompanying risk factors. As clinicians are confronted with an increasing number of non-albicans Candida species, knowledge about these pathogens and their sensitivities is of major importance.

Keywords: Children, Candidiasis, Non-albicans Candida species, Urinary tract infection

\section{Background}

Systemic Candida infections in children are of major concern in preterm infants, neonates with risk factors and in immunocompromised children [1,2]. Further risk factors such as use of central venous catheters, longer use of broad spectrum antibiotics and use of parenteral nutrition contribute as well [2]. Over the last decade non-albicans Candida species are emerging as causative pathogens for systemic Candida infections in children $[3,4]$. Here, we report of a candidiasis caused by Candida kefyr in a term neonate.

\footnotetext{
* Correspondence: Stefan.Weichert@medma.uni-heidelberg.de 'Pediatric Infectious Diseases, University Children's Hospital Mannheim of Heidelberg University, Mannheim, Germany

Full list of author information is available at the end of the article
}

\section{Case Presentation}

After an uneventful birth anal atresia was observed and a vesico-ureteral-reflux (VUR) grade $\mathrm{V}$ was detected by cystography. Renal agenesis on the left side was already diagnosed antenatally. The first surgical procedure, creating a protective colostoma, on day 2 was uneventful. The child was treated with intravenous cefotaxime for 10 days and was put hereafter on cefixime prophylaxis. On day 21 the patient developed an urosepsis caused by Enterococcus faecalis which was treated with piperacillin according to the antibiogramm. After initial improvement the child deteriorated again 10 days after initiation of antibiotic treatment. Antibiotic therapy was changed to imipenem, gentamicin and vancomycin. A lumbar puncture showed normal results, but the urine analysis revealed significant fungal growth $\left(10^{6} \mathrm{CFU} /\right.$

\section{Biomed Central}


$\mathrm{mL}$ ). Primary isolation was performed on Candida Chrom $^{\text {TM }}$ Agar (BD) which yielded growth of large rough pink colonies, resembling Candida krusei. Therefore systemic antifungal therapy was initiated with liposomal amphotericin B (AMB). Further identification of the suspected non-albicans Candida species was performed by biochemical identification using the API $20 \mathrm{C}$ AUX (BioMerieux) biochemical identification panel which yielded excellent identification (probability > 99.9\%, profile number 7220300031 ). This biochemical result was confirmed by sequencing of the internal transcribed spacer (ITS) regions using primer pairs ITS 1 and ITS-4 (ITS1: 5-TCCGTAGGTGAACCTGCGG-3, ITS4: 5-TCCTCCGCTTATTGATATGC-3 and V9D:5TTAAGTCCCTGCCCTTTGTA-3 and LS266:5GCATTCCCAAACAACTCGACTC-3, respectively) [5]. Both primers span the complete ITS1, 5.8S, and ITS2 regions. A databank search of the amplified sequences revealed $99 \%$ and 100\% homology with the ITS region from Kluyveromyces marxianus which represents the teleomorph form of Candida kefyr. Susceptibility testing against fluconazole, amphotericin B (AMB), and caspofungin was performed by ellipsometer test ("E-test") and showed a minimal inhibitory concentrations (MIC) of $0,25 \mu \mathrm{g}, 0,047 \mu \mathrm{g}$, and 0,25 $\mu \mathrm{g}$, respectively. All tests were repeated two times with similar results. The inoculum for susceptibility testing was generally performed by pooling of 10-20 individual colonies. No macrocolonies were observed in the inhibition zone of the E-test. Further subplating and antibiotic susceptibility testing of individual colonies in order to detect antibiotic susceptibility variants was not performed. Species-specific susceptibility breakpoints for Candida kefyr have neither been published by the clinical laboratory institute (CLSI) nor by the European committee on antimicrobial susceptibility testing (EUCAST). Therefore we used the EUCAST breakpoints for Candida albicans for the interpretation of the MICs obtained with the Candida kefyr isolate. According to these breakpoints the isolate was susceptible to all three antifungal agents tested. After the availability of susceptibility data antifungal treatment was changed to fluconazole. Although the child improved clinically, a significant candiduria persisted and renal ultrasound showed persistent signs of Candida pyelonephritis (Figure 1). Blood culture results turned out to be negative. An initial contrast enema did not show a connecting fistula between bladder and rectum. But due to the clinical course a fistula was suspected and surgical repair of the anorectal atresia was performed. While undergoing surgery (creating a neo-rectum) a recto-vesical fistula was found and subsequently was resected. Since high grade reflux was prevalent in our patient AMB $(1 \mu \mathrm{g} / \mathrm{mL})$ was successfully instilled into the child's bladder twice daily over 7 days. Candida kefyr was

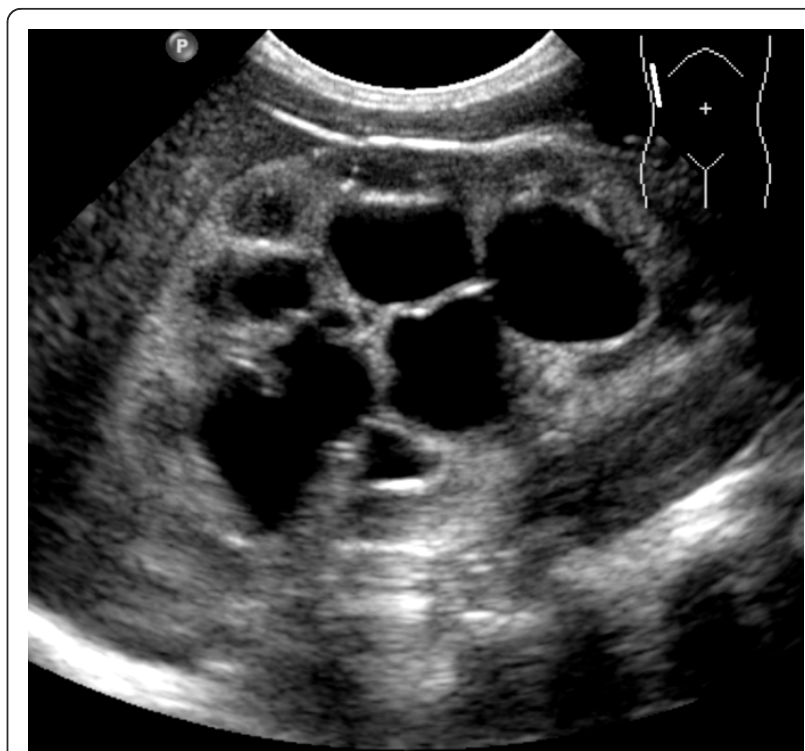

Figure 1 Renal ultrasound. Hyperechogenic renal parenchyma due to persistent Candida pyelonephritis and massive pelviectasis.

isolated for the last time after 7 days of treatment, afterwards, all tested urine cultures remained sterile and a second blood culture remained sterile as well. The child recovered completely under systemic fluconazole therapy ( $8 \mathrm{mg} / \mathrm{kg} /$ day) over 3 months.

\section{Discussion}

The high burden of systemic Candida infection in children with risk factors led to a significant increase in fluconazole use over the last decades, which was accompanied by an increased incidence of non-albicans Candida species. Interestingly, susceptibility of the main causative pathogen Candida albicans to fluconazole remains stable $[3,4]$. In contrast, a recent study showed only $82 \%$ susceptibility of all isolated non-albicans Candida species to fluconazole [3]. Data regarding susceptibilities of antifungal agents against Candida kefyr are limited. The isolated Candida kefyr from our patient was fully sensitive to fluconazole. In a 10.5-year worldwide surveillance study resistance to fluconazole ranged from $3.3 \%$ in the first 4 study years to $1.7 \%$ for all Candida kefyr isolates in the last 3 study-years [4]. So far, good susceptibilities of AMB against most non-albicans Candida species were shown, although country specific differences were observed [4,6,7]. According to a study from Pfaller et al. the susceptibility of Candida kefyr to amphotericin B appears to be quite low (4 of 10 isolates were susceptible at $\leq 1 \mu \mathrm{g} / \mathrm{ml}$ ) [8]. A study conducted in Germany involving mainly adult patients showed an increased MIC of AMB for 9\% of all Candida kefyr isolates [9], whereas a more recent study from Spain showed no increased MIC of AMB [7]. 
Although our patient had recurrent infections due to Candida kefyr and had clinical symptoms of systemic disease the pathogen Candida kefyr was only isolated from urine cultures and not from blood cultures or other sites. Our patient suffered from grade V reflux, that may led to an ascending kidney infection. However, it is reported that amongst clinical signs for systemic disease isolated candiduria may be the only indication for candidaemia. Studies confirmed that blood cultures are $40-75 \%$ false negative in patients with candidiasis, as demonstrated in patients with autopsy proven candidiasis $[10,11]$. In addition to clinical signs of systemic disease, our patient had renal involvement as well, such as parenchymal changes on ultrasound. An ascending infection would be expected to result in isolated pelvicalyceal disease, and it is known that haematogenous spread is the most common route for renal candidiasis [12]. Therefore, it is conceivable, that patients may have transient candidaemia that may lead to organ involvement. Nevertheless, it is known that blood cultures are often no longer positive when renal candidiasis becomes manifest [13]. As candiduria is regarded as a risk factor for invasive candidiasis [14] clinicians should be aware of this, even though blood cultures might remain negative.

Up to now Candida kefyr is considered as not pathogenic to healthy individuals, but has been discussed as an emerging pathogen in patients with risk factors. Pediatric data are sparse, reporting isolation of Candida kefyr from $1.8 \%$ to $4 \%$ of all isolated Candida species from mainly preterm und low birth weight neonates $[15,16]$. In adults Candida kefyr has been reported to cause systemic Candida infection in patients with neutropenic leukemia [17] and in a woman with underlying heart disease [18]. Very recently Candida kefyr was described as a pathogen causing invasive fungal enteritis in a patient with underlying haematological disease following bone marrow transplantation [19]. Of note, Sendid et al. report a twofold detection rate of Candida kefyr isolates from adult patients in oncohematology wards compared to patients in other wards $(4.8 \%$ vs. 1.9\%) [20]. Up to now, it is not known why Candida kefyr is found more often in these patients. Induced selection of Candida kefyr following antimicrobial therapy or prophylaxis is discussed, as well as factors that might influence gastrointestinal homeostasis in favour of Candida kefyr [20]. Furthermore, as Candida kefyr is commonly found in dairy products, dietary habits might influence or promote colonization and subsequent infection in patients as well [21].

\section{Conclusion}

As clinicians are confronted with an increasing number of non-albicans Candida species, knowledge about these pathogens and their sensitivities is of major importance. In children with recurrent candiduria systemic infection and organ involvement should be ruled out, even though blood cultures might remain negative.

\section{Consent}

Written informed consent was obtained from the patient's guardian for publication of this case report and any accompanying images. A copy of the written consent is available for review by the Editor-in-Chief of this journal.

\section{Author details \\ ${ }^{1}$ Pediatric Infectious Diseases, University Children's Hospital Mannheim of Heidelberg University, Mannheim, Germany. ${ }^{2}$ Department of Pediatric Surgery, University Hospital Mannheim of Heidelberg University, Mannheim, Germany. ${ }^{3}$ Institute for Medical Microbiology and Hygiene, University Hospital Mannheim of Heidelberg University, Mannheim, Germany. ${ }^{4}$ Department of Clinical Radiology and Nuclear Medicine, University Hospital Mannheim of Heidelberg University, Mannheim, Germany. ${ }^{5}$ Pediatric Infectious Diseases, University Children's Hospital Mannheim of Heidelberg University, Theodor-Kutzer-Ufer 1-3, 68167 Mannheim, Germany.}

\section{Authors' contributions}

SW took care of the patient and drafted and wrote the manuscript. KR took care of the patient and contributed to the draft of the manuscript. KZ took care of the patient and helped to the draft of the manuscript. GG performed and interpreted all mentioned microbiological methods and revised the manuscript. AD performed and confirmed identification of the mentioned pathogen. AK performed the ultrasound imaging studies and contributed to the draft of the manuscript. HS contributed in coordinating the manuscript and to the draft of the manuscript. $\Pi T$ took care of the patient and coordinated and edited the manuscript. All authors have read the manuscript and approved its final version.

\section{Competing interests}

The authors declare that they have no competing interests.

Received: 4 September 2011 Accepted: 18 March 2012

Published: 18 March 2012

\section{References}

1. Zaoutis TE, Greves HM, Lautenbach E, Bilker WB, Coffin SE: Risk factors for disseminated candidiasis in children with candidemia. Pediatr Infect Dis J 2004, 23:635-641.

2. Manzoni P, Stolfi I, Pugni L, Decembrino L, Magnani C, Vetrano G, Tridapalli E, Corona G, Giovannozzi C, Farina D, Arisio R, Merletti F, Maule M, Mosca F, Pedicino R, Stronati M, Mostert M, Gomirato G: A multicenter, randomized trial of prophylactic fluconazole in preterm neonates. $N$ Engl J Med 2007, 356:2483-2495.

3. Neu N, Malik M, Lunding A, Whittier S, Alba L, Kubin C, Saiman L: Epidemiology of candidemia at a Children's hospital, 2002 to 2006. Pediatr Infect Dis J 2009, 28:806-809.

4. Pfaller MA, Diekema DJ, Gibbs DL, Newell VA, Ellis D, Tullio V, Rodloff A, Fu W, Ling TA: Results from the ARTEMIS DISK Global Antifungal Surveillance Study, 1997 to 2007: a 10.5-year analysis of susceptibilities of Candida Species to fluconazole and voriconazole as determined by CLSI standardized disk diffusion. J Clin Microbiol 2010, 48:1366-1377.

5. White TJ, Bruns T, Lee S, Taylor JW: Amplification and direct sequencing of fungal ribosomal RNA genes for phylogenetics. In PCR Protocols: A Guide to Methods and Applications. Edited by: Innis MA, Gelfand DH, Sninsky JJ, White TJ. New York: Academic; 1990:315-322.

6. Tortorano AM, Peman J, Bernhardt H, Klingspor L, Kibbler CC, Faure O, Biraghi E, Canton E, Zimmermann K, Seaton S, Grillot R: Epidemiology of candidaemia in Europe: results of 28-month European Confederation of 
Medical Mycology (ECMM) hospital-based surveillance study. Eur I Clin Microbiol Infect Dis 2004, 23:317-322

7. Gomez-Lopez A, Pan D, Cuesta I, Alastruey-Izquierdo A, Rodriguez-Tudela JL, Cuenca-Estrella M: Molecular identification and susceptibility profile in vitro of the emerging pathogen Candida kefyr. Diagn Microbiol Infect Dis 2010, 66:116-119.

8. Pfaller MA, Diekema DJ, Messer SA, Boyken L, Hollis RJ, Jones RN: In vitro activities of voriconazole, posaconazole, and four licensed systemic antifungal agents against Candida species infrequently isolated from blood. J Clin Microbiol 2003, 41:78-83.

9. Borg-von Zepelin M, Kunz L, Rüchel R, Reichard U, Weig M, Gross U: Epidemiology and antifungal susceptibilities of Candida spp. to six antifungal agents: results from a surveillance study on fungaemia in Germany from July 2004 to August 2005. J Antimicrob Chemother 2007, 60:424-428.

10. Mirdha BR, Sethi S, Banerjee U: Prevalence of fungal species in patients with candiduria. Indian J Med Res 1998, 107:90-93.

11. Ostrosky-Zeichner L, Pappas PG: Invasive candidiasis in the intensive care unit. Crit Care Med 2006, 34:857-863.

12. Robinson JL, Davies HD, Barton M, O'Brien K, Simpson K, Asztalos E, Synnes A, Rubin E, Le Saux N, Hui C, Langley JM, Sauve R, de Repentigny L, Kovacs L, Tan B, Richardson SE: Characteristics and outcome of infants with candiduria in neonatal intensive care - a Paediatric Investigators Collaborative Network on Infections in Canada (PICNIC) study. BMC Infect Dis 2009, 9:183.

13. Lundstrom T, Sobel J: Nosocomial candiduria: a review. Clin Infect Dis 2001, 32:1602-1607.

14. Olaechea PM, Palomar M, Leon-Gil C, Alvarez-Lerma F, Jordá R, Nolla-Salas J, León-Regidor MA, EPCAN Study Group: Economic impact of candida colonization and candida infection in the critically ill patient. Eur J Clin Microbiol Infect Dis 2004, 23:323-330.

15. Altuncu E, Bilgen H, Cerikçioğlu N, Ilki A, Ulger N, Bakır M, Akman I, Ozek E: Neonatal Candida infections and the antifungal susceptibilities of the related Candida species. Mikrobiyol Bul 2010, 44:593-603.

16. Rani R, Mohapatra NP, Mehta G, Randhawa VS: Changing trends of Candida species in neonatal septicaemia in a tertiary North Indian hospital. Indian J Med Microbiol 2002, 20:42-44.

17. Reuter CW, Morgan MA, Bange FC, Gunzer F, Eder M, Hertenstein B, Ganser A: Candida kefyr as an emerging pathogen causing nosocomial bloodstream infections in neutropenic leukemia patients. Clin Infect Dis 2005, 41:1365-1366.

18. Chopra T, Bhargava A, Kumar S, Chopra A, Dhar S, Afonso L, Sobel JD: Candida kefyr endocarditis in a patient with hypertrophic obstructive cardiomyopathy. Am J Med Sci 2010, 339:188-189.

19. Direkze S, Mansour M, Rodriguez-Justo M, Kibbler C, Gant V, Peggs KS: Candida kefyr fungal enteritis following autologous BMT. Bone Marrow Transplantation 2011, doi:10.1038/bmt.2011.112.

20. Sendid B, Lacroix C, Bougnoux ME: Is Candida kefyr an emerging pathogen in patients with oncohematological diseases? Clin Infect Dis 2006, 43:666-667.

21. Valderrama MJ, de Silóniz MI, Gonzalo P, Peinado JM: A differential medium for the isolation of Kluyveromyces marxianus and Kluyveromyces lactis from dairy products. J Food Prot 1999, 62:189-193.

\section{Pre-publication history}

The pre-publication history for this paper can be accessed here: http://www.biomedcentral.com/1471-2334/12/61/prepub

doi:10.1186/1471-2334-12-61

Cite this article as: Weichert et al:: Candidiasis caused by Candida kefyr in a neonate: Case report. BMC Infectious Diseases 2012 12:61.

\section{Submit your next manuscript to BioMed Central and take full advantage of:}

- Convenient online submission

- Thorough peer review

- No space constraints or color figure charges

- Immediate publication on acceptance

- Inclusion in PubMed, CAS, Scopus and Google Scholar

- Research which is freely available for redistribution

Submit your manuscript at www.biomedcentral.com/submit
Biomed Central 